\title{
Quasiconformal mappings of $Y$-pieces
}

Christopher J. Bishop

\begin{abstract}
In this paper we construct quasiconformal mappings between $Y$-pieces so that the corresponding Beltrami coefficient has exponential decay away from the boundary. These maps are used in a companion paper to construct quasiFuchsian groups whose limit sets are non-rectifiable curves of dimension 1.
\end{abstract}

\section{Introduction}

The purpose of this note is to record an explicit way of deforming a Riemann surface so as to shorten a given closed geodesic, with a careful estimate of the complex dilatation $\mu$ of the corresponding map. In particular, we will do this so that $\mu$ has the optimal $L^{\infty}$ norm (up to a bounded factor) and $|\mu|$ decays exponentially fast away from $\gamma$. This estimate is used in the companion paper [3] to construct quasiFuchisan groups whose limit sets are nonrectifiable curves of dimension 1.

A generalized $Y$-piece is a Riemann surface (with boundary) which is bounded by three closed geodesics (or punctures) and which is homeomorphic to a 2-sphere minus three disks (or points). It is called $L$ bounded if each of the boundary components has length $\leq L$ (punctures count as length zero). Every finite area Riemann surface can be written as a finite union of such pieces and even general Riemann surfaces can be written as a union of $Y$-pieces, funnels and half-planes, [1].

2000 Mathematics Subject Classification: Primary: 30F60.

Keywords: Hausdorff dimension, quasi-Fuchsian groups, quasiconformal deformation, critical exponent, convex core. 
Theorem 1.1. Suppose $Y_{1}$ is an $L$ bounded $Y$-piece with boundary components $\gamma_{a}, \gamma_{b}$ and $\gamma_{c}$ with lengths $\left(a_{1}, b_{1}, c_{1}\right)$ respectively. Suppose $Y_{2}$ is another $L$-bounded generalized $Y$-piece with boundary lengths $\left(a_{2}, b_{1}, c_{1}\right)$ respectively. Assume that $\epsilon=\left|\log a_{1} / a_{2}\right| \leq 2$. Then there is a $C=C(L)$ and a quasiconformal map $f: Y_{1} \rightarrow Y_{2}$ with constant $K \leq 1+C \epsilon$ which is affine on each of the boundary components (i.e. is isometric on $\gamma_{b}$ and $\gamma_{c}$ and multiplies length by $a_{2} / a_{1}$ on $\gamma_{a}$ ). Moreover, the Beltrami coefficient $\mu$ of $f$ satisfies

$$
|\mu(z)| \leq C \epsilon \exp \left(-2 \operatorname{dist}\left(z, \gamma_{a}\right)\right)
$$

Thus the Beltrami coefficient $\mu$ has $L^{\infty}$ norm which is comparable to the optimal quasiconformal map from $Y_{1}$ to $Y_{2}$, but has rapid decay away from boundary curve which is being shrunk. This is important for our applications in [3] where we wish to estimate integrals involving $\mu$.

An $X$-piece is the union of two (not necessarily distinct) $Y$-pieces which share a common boundary geodesic $\gamma$, which is called the central curve of $X$. As above an $X$-piece is called $L$-bounded if its boundary components and central curve all have length bounded by $L$. The following is an obvious consequence of the theorem and is the precise result used in [3].

Corollary 1.2. Given an L-bounded $X$-piece $X_{1}$, its central curve $\gamma$ and any $0<\epsilon<1 / 2$, there is an $X$-piece $X_{2}$ and a quasiconformal map $f: X_{1} \rightarrow X_{2}$ so that $f(\gamma)$ is the central geodesic for $X_{2}$ and has length $1-\epsilon$ times that of $\gamma$. Moreover, $f$ is an isometry on the boundary of $X_{1}$ and the corresponding Beltrami coefficient $\mu_{\gamma, \epsilon}$ satisfies $\left\|\mu_{\gamma, \epsilon}\right\|_{\infty}<C \epsilon$ and

$$
\left|\mu_{\gamma, \epsilon}(z)\right| \leq C \epsilon \exp (-2 \operatorname{dist}(z, \gamma)) \text {. }
$$

Using this we can construct a deformation of Riemann surface $R$ by choosing an $X$-piece $X_{1}$ in $R$ and replacing it by another $X$-piece $X_{2}$, to get a surface $S$. We then define a map $R \rightarrow S$ by taking the identity off $X_{1}$ and the map from the corollary on $X_{1}$. The deformations constructed in [3] are obtained by applying this idea simultaneously to certain infinite (disjoint) collections of $X$-pieces in $R$.

Other applications of our estimate are given in [6] and [7]. Let $\mathcal{T}$ denote the set of Riemann surfaces which can be written as a countable union of $Y$-pieces with the property that for any $\epsilon>0$, all but finitely many of the pieces are $\epsilon$ bounded. In [7] our estimate used to show that Fuchsian groups corresponding to surfaces in $\mathcal{T}$ are $\delta$-stable, i.e., for any quasiconformal deformation of the group $\delta=\operatorname{dim}(\Lambda)$, i.e. the critical exponent equals the Hausdorff dimension of the limit set. In [6], another application is given related to Ruelle's property. See [4] and [6]. 
Next we describe the structure of the rest of the paper.

Section 2: We review some hyperbolic trigonometry.

Section 3: We show that two hyperbolic triangles are quasiconformally equivalent with constant close to 1 if the ratio of their side lengths is close to 1 .

Section 4: We construct quasiconformal maps between certain hyperbolic quadrilaterals.

Section 5: We show that certain hyperbolic hexagons can be written as a union of a bounded piece and at most three quadrilaterals.

Section 6: We prove Theorem 1.1.

I thank Michel Zinsmeister for his comments and questions on [5] which led me to consider the questions dealt with here. I also thank the two referees whose careful reading and numerous suggestions were greatly appreciated.

If $A, B$ are quantities that depend on some parameter we write $A \lesssim B$ if the ratio $B / A$ is bounded uniformly independent of the parameter. We will also sometimes write this as $A=O(B)$. Similarly for $\gtrsim$. We write $A \simeq B$ if both $A \lesssim B$ and $A \gtrsim B$ hold and say $A$ and $B$ are comparable.

\section{Some hyperbolic trigonometry}

In this section we record a few formulas of hyperbolic trigonometry which we will need later.

Recall the sine and cosine rules for hyperbolic geometry (e.g., see page 148 of Beardon's book [2]). Let $T$ denote a hyperbolic triangle with angles $\alpha, \beta, \gamma$ and opposite side lengths denoted by $a, b, c$. See Figure 1.

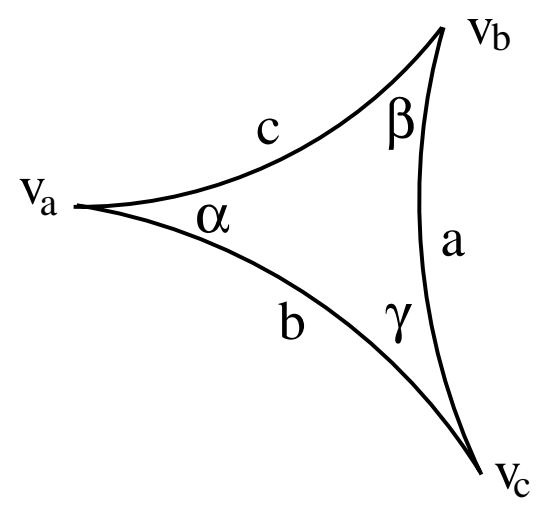

Figure 1: Definitions of $a, b, c$ and $\alpha, \beta, \gamma$ 
Then we have the Sin Rule,

$$
\frac{\sinh a}{\sin \alpha}=\frac{\sinh b}{\sin \beta}=\frac{\sinh c}{\sin \gamma}
$$

the First Cosine Rule,

$$
\cosh c=\cosh a \cosh b-\sinh a \sinh b \cos \gamma
$$

and the Second Cosine Rule

$$
\cosh c=\frac{\cos \alpha \cos \beta+\cos \gamma}{\sin \alpha \sin \beta}
$$

Lemma 2.1. If $\gamma=\pi / 2$ then

$$
\begin{aligned}
\sinh b & =\sinh c \sin \beta, \\
\tanh b & =\sinh a \tan \beta, \\
\tanh a & =\tanh c \cos \beta .
\end{aligned}
$$

Proof. The first of these is immediate by taking $\gamma=\pi / 2$ in (2.1). To prove (2.5), note that

$$
\begin{aligned}
\tanh b=\frac{\sinh b}{\cosh b} & =\frac{\sin \beta}{\sin \alpha} \frac{\sinh a}{\cosh b} \\
& =\frac{\sin \beta}{\sinh a \cosh a} \frac{\cosh c}{\sin \beta} \sinh a \cosh a \frac{\sin \alpha \sin \beta}{\cos \alpha \cos \beta} \\
& \left.=\frac{\sin \alpha}{\sin \beta}\right] \\
& =\tan \beta \sinh a\left[\cosh a \frac{\cos \alpha}{\sin }\right] \\
& =\tan \beta \sinh a .
\end{aligned}
$$

where the second, third and fourth equalities hold by (2.1), (2.2) and (2.3) respectively and the last equality holds by (2.3) (with the roles of $a$ and $c$ reversed). To prove (2.6), note that

$$
\begin{aligned}
\tanh a=\frac{\sinh a}{\cosh a} & =\frac{\sinh a \cosh b}{\cosh c} \\
& =\frac{\tanh b \cos \beta \cosh b}{\sin \beta \cosh c} \\
& =\tanh c \cos \beta\left[\frac{\sinh b}{\sinh c \sin \beta}\right] \\
& =\tanh c \cos \beta,
\end{aligned}
$$

where the second, third and last equalities hold by (2.2), (2.5) and (2.1) respectively. 
Formula (2.3) makes it clear that the side lengths of a hyperbolic triangle are determined by its angles. It is also easy to see that if all three angles are bounded below by some $\theta>0$, then there is a finite upper bound on the side lengths which only depends on $\theta$. By (2.3), if all three angles of a hyperbolic triangle are less than $\delta$ the the diameter of the triangle is $\geq M(\delta)$ where $M \rightarrow \infty$ as $\delta \rightarrow 0$.

By (2.1), if the three sides of a hyperbolic triangle have comparable lengths then the sin of the angles are also comparable. Since at most one of the angles can be close to $\pi$ we see that if all the angles are bounded away from $\pi$ then they must all be comparable in size.

\section{Quasiconformal mappings of hyperbolic triangles}

In this section we record a calculation which we will need later. It simply says that two hyperbolic triangles whose side lengths are pairwise close are quasiconformally equivalent with constant close to 1 . This seems fairly obvious, but I have been unable to locate the result in the literature, so it seems worth recording here. Our strategy will be to divide the triangles into small triangles which are approximately Euclidean and then apply the analogous result for Eucidean trangles (which is easy).

Lemma 3.1. Suppose $T_{1}, T_{2} \subset \mathbb{D}$ are two triangles in the hyperbolic disk with angles $\left(\alpha_{1}, \beta_{1}, \gamma_{1}\right)$ and $\left(\alpha_{2}, \beta_{2}, \gamma_{2}\right)$ respectively and opposite sides $\left(a_{1}, b_{1}, c_{1}\right)$ and $\left(a_{2}, b_{2}, c_{2}\right)$ respectively. Suppose that all these angles are strictly positive, say all $\geq \theta>0$ and let

$$
\epsilon=\max \left(\left|\log \frac{a_{1}}{a_{2}}\right|,\left|\log \frac{b_{1}}{b_{2}}\right|,\left|\log \frac{c_{1}}{c_{2}}\right|\right)
$$

Suppose $\epsilon \leq A$. Then there is a constant $C_{1}=C_{1}(\theta, A)$ and a $1+C_{1} \epsilon$ quasiconformal map from $T_{1}$ to $T_{2}$ which maps each vertex to the corresponding vertex and which is affine on each edge of $T_{1}$ (i.e., it multiplies hyperbolic arclength on each edge by the appropriate factor $a_{2} / a_{1}, b_{2} / b_{1}$ or $\left.c_{2} / c_{1}\right)$.

Proof. The angle bisectors of each of the three angles of $T_{1}$ meet at a single point $\zeta_{1}$ in the interior of $T_{1}$ by Theorem 7.14.1 of [2]. See Figure 2.

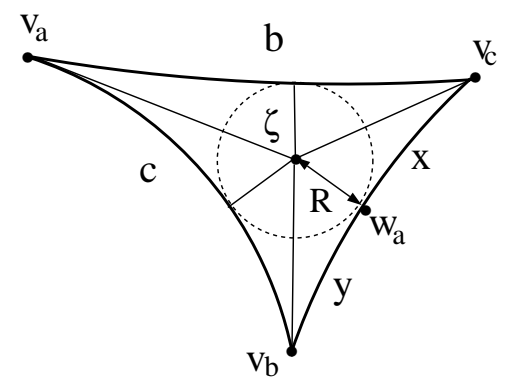

Figure 2: Angle bisectors meet at a point 
This point is equidistant from each of there three sides of $T_{1}$. Let $R_{1}$ denote this distance and let $w_{a}^{1}, w_{b}^{1}, w_{c}^{1}$ denote the closest point to $\zeta_{1}$ on each of the sides $a_{1}, b_{1}, c_{1}$ respectively. Divide each edge of $T_{1}$ into $2^{n}$ equal length segments and connect the endpoints of these segments by geodesics segments to $\zeta_{1}$. This divides $T_{1}$ into $3 \cdot 2^{n}$ sub-triangles $\left\{T_{1, k}\right\}$. See Figure 3 .

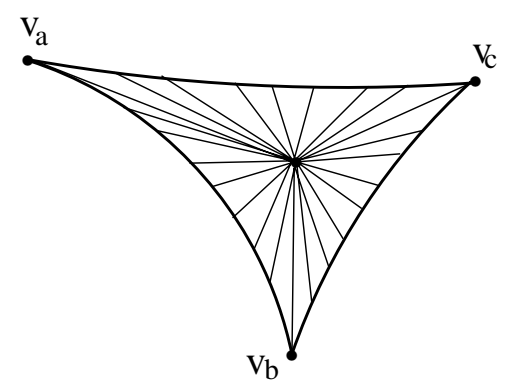

Figure 3: The triangles $T_{k}$ 's

Assume $\zeta_{1}=0$ and for each $k=1, \ldots, 3 \cdot 2^{n}$ let $\tilde{T}_{1, k}$ be the Euclidean triangle which has the same vertices as $T_{1, k}$ (since $\zeta_{1}=0$, two sides of $\tilde{T}_{1, k}$ will agree exactly with two sides of $T_{1, n}$ but the third side opposite $\zeta_{1}$ will not). Let $\zeta_{2}, R_{2},\left\{T_{2, k}\right\}$ and $\left\{\tilde{T}_{2, k}\right\}$ be the corresponding objects for $T_{2}$ and assume $\zeta_{2}=0$ as well.

We claim that there is a $C=C(\theta)<\infty$ so that each $\tilde{T}_{1, k}$ can be mapped to $\tilde{T}_{2, k}$ by a $1+C \epsilon$ quasiconformal map which is affine in the Euclidean sense. If so, then adjacent triangles have matching boundary values so we get a $1+C \epsilon$ quasiconformal from $\cup_{k} \tilde{T}_{1, k}$ to $\cup_{k} \tilde{T}_{2, k}$. Then taking $n \rightarrow \infty$ we obtain in the limit a $1+C \epsilon$ quasiconformal map from $T_{1}$ to $T_{2}$. By definition this map is affine restricted to the vertices of $E \cap \cup_{k} \tilde{T}_{1, k}$ for each edge $E$ of $T_{1}$, and so by continuity is affine on each edge, as desired. Thus it suffices to verify the claim.

Let $L_{1}$ denote an edge of $\tilde{T}_{1, k}$ which connects $\zeta_{1}$ to an edge $E_{1}$ of $T_{1}$. Let $\theta_{1}$ be the angle formed by $L_{1}$ and $E_{1}$ and let $F_{1}$ be the edge of $\tilde{T}_{1, k}$ which is opposite $\zeta_{1}$. It is clear that $\theta_{1}$ is minimized when $L_{1}$ is one of the angle bisectors of $T_{1}$ and hence $\frac{1}{2} \theta \leq \theta_{1} \leq \frac{1}{2} \pi$ (where $\theta$ is assumed the lower bound on the angles of $T_{1}$ ). Let $L_{2}, E_{2}, F_{2}$ and $\theta_{2}$ be the corresponding quantities in $T_{2}$. It is enough to check that the Euclidean lengths of these edges satisfy

$$
\begin{aligned}
\left|L_{1}\right| /\left|L_{2}\right| & =1+O(\epsilon), \\
\left|F_{1}\right| /\left|F_{2}\right| & =1+O(\epsilon), \\
\theta_{1} / \theta_{2} & =1+O(\epsilon) .
\end{aligned}
$$

(It is easy to check that Euclidean triangles satisfying these conditions are $1+O(\epsilon)$ quasiconformally equivalent via an affine map.) 
We start by showing $R_{1} / R_{2}=1+O(\epsilon)$. Let $x_{i}=\rho\left(w_{a}^{i}, v_{c}^{i}\right)$ and $y_{i}=$ $\rho\left(w_{a}^{i}, v_{b}^{i}\right)$ so that $x_{i}+y_{i}=a_{i}$ (see Figure 4$)$.

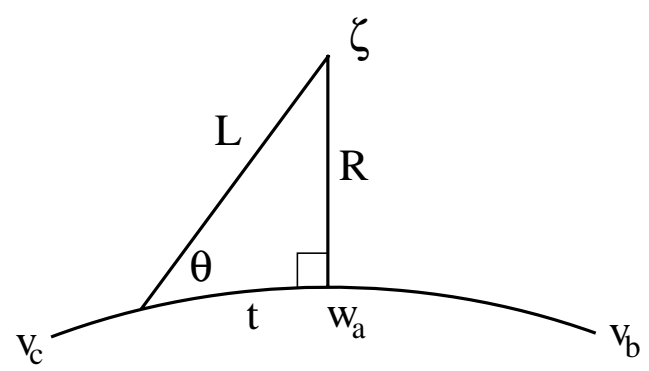

Figure 4: Definition of $L_{i}$ and $R_{i}$

Using (2.5) we see that

$$
\tanh R_{i}=\sinh x_{i} \tan \left(\alpha_{i} / 2\right)=\sinh y_{i} \tan \left(\beta_{i} / 2\right) .
$$

Since

$$
\frac{x_{1}+y_{1}}{x_{2}+y_{2}}=1+O(\epsilon)
$$

we must have either $x_{1} / x_{2} \geq 1-O(\epsilon)$ or $y_{1} / y_{2} \geq 1-O(\epsilon)$. Without loss of generality assume the first holds. Then

$$
\frac{\tanh R_{1}}{\tanh R_{2}}=\frac{\sinh x_{1} \tan \left(\alpha_{1} / 2\right)}{\sinh x_{2} \tan \left(\alpha_{2} / 2\right)} \geq 1-O(\epsilon)
$$

Since $R_{i}, x_{i}$ are uniformly bounded (in terms of $\theta$ ) and $\alpha_{i}$ is bounded away from $\pi$ (depending only on $\theta$ ), this implies that $R_{1} / R_{2} \geq 1-O(\epsilon)$ where the constant depends only on $\theta$. Reversing the roles of $R_{1}$ and $R_{2}$ shows $R_{1} / R_{2}=1+O(\epsilon)$, as desired.

Next we prove (3.1). Suppose $L_{i}$ connects $\zeta_{i}$ to the edge $a_{i}$ of $T_{i}$ and consider the hyperbolic triangle with edge $L_{i}$ and third vertex $w_{a}^{i}$. See Figure 4 . Let $t_{i}$ be the signed hyperbolic distance from $w_{a}^{i}$ to the endpoint of $L_{i}$ on the edge $a_{i}$. Note that $\left|t_{1}-t_{2}\right|=O\left(\epsilon a_{1}\right)$. Then by $(2.2)$, with $\gamma=\pi / 2$,

$$
\cosh L_{i}=\cosh t_{i} \cosh R_{i} .
$$

Now for $0 \leq s \leq 1$ let $t(s)=t_{1}(1-s)+t_{2} s, R(s)=R_{1}(1-s)+R_{2} s$ and $\cosh L(s)=\cosh t(s) \cosh R(s)$. Then clearly

$$
\left|t^{\prime}(s)\right|=O\left(\epsilon a_{1}\right), \quad\left|R^{\prime}(s)\right|=O\left(\epsilon a_{1}\right),
$$


and

$$
\begin{aligned}
\left|L^{\prime}(s)\right| & \leq \frac{1}{\sinh L(s)}\left[\sinh t(s) t^{\prime}(s) \cosh R(s)+\cosh t(s) \sinh R(s) R^{\prime}(s)\right] \\
& =O\left(\epsilon a_{1}\right) .
\end{aligned}
$$

Thus $\left|L_{1}-L_{2}\right|=O\left(\epsilon a_{1}\right)=O\left(\epsilon L_{1}\right)$. Thus $\left|L_{1}\right| /\left|L_{2}\right|=1+O(\epsilon)$, as desired.

Now we prove (3.2). The segments $F_{1}, F_{2}$ have hyperbolic diameters which are comparable by a factor of $1+O(\epsilon)$, since by definition they have hyperbolic length $2^{-n} a_{1}$ and $2^{-n} a_{2}$ and $a_{1} / a_{2}=1+O(\epsilon)$ by assumption. Moreover, their hyperbolic distances from the origin are $L_{1}$ and $L_{2}$ which are comparable by the previous paragraph. Since $|d z|=\left(1-|z|^{2}\right)|d \rho|$ this implies their Euclidean lengths are also comparable by a factor of $1+O(\epsilon)$.

Finally we prove (3.3). By (2.6),

$$
\cos \theta_{i} \tanh L_{i}=\tanh t_{i}
$$

and hence making a parameterized family as above we see

$$
\sin \theta(s) \theta^{\prime}(s) \tanh L(s)+\cos \theta(s) \operatorname{sech}^{2} L(s) L^{\prime}(s)=\operatorname{sech}^{2} t(s) t^{\prime}(s),
$$

and since $\theta(s)$ is bounded away from zero in terms of $\theta$, and $\left|t^{\prime}(s)\right|,\left|L^{\prime}(s)\right|=$ $O(\epsilon a)$, we get

$$
\left|\theta^{\prime}(s)\right|=O(1) \frac{1}{\tanh L(s)}\left|\operatorname{sech}^{2} t(s)-\cos \theta \operatorname{sech}^{2} L(s)\right| O(\epsilon a)=O(\epsilon) .
$$

Thus $\left|\theta_{1}-\theta_{2}\right|=O(\epsilon)$ and since these numbers are bounded by $\theta$, we get $\theta_{1} / \theta_{2}=1+O(\epsilon)$, with constant depending only on $\theta$. This ends the proof of Lemma 3.1.

The preceding lemma can be used to construct quasiconformal maps between polygons by triangulating the polygon and applying the lemma to each piece. Since the maps are affine on the edges, they must agree on the common boundary of any two triangles which share an edge. The following corollaries describe two specific situations where we will use this.

Corollary 3.2. Suppose $Q$ is a hyperbolic quadrilateral with sides $a_{1}, a_{2}, a_{3}$ and $a_{4}$ (in clockwise direction) all bounded by $L<\infty$. Suppose the two angles adjacent to edge $a_{1}$ are right angles and the other two angles are bounded below by $\theta>0$ and above by $\pi-\theta$. Suppose $Q^{\prime}$ is another quadrilateral with side lengths $b_{1}, b_{2}, b_{3}, b_{4}$ whose angles satisfy the same condition. Let $\epsilon=\max _{i}\left|\log b_{i} / a_{i}\right|$ and assume $\epsilon<A<\infty$. Then there is a $1+C(L, \theta, A) \epsilon$ quasiconformal map from $Q$ to $Q^{\prime}$ which is affine on each of the boundary edges. 
Proof. Connect two opposite corners of $Q$ forming two triangles with edge lengths $a_{1}, a_{2}, c$ and $a_{3}, a_{4}, c$. See Figure 5 .

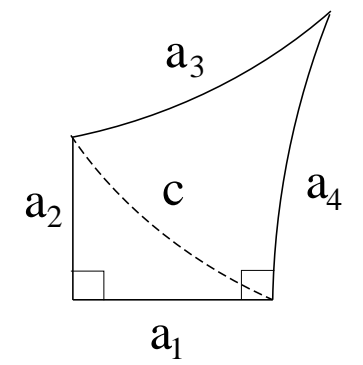

FiguRE 5

By (2.2),

$$
\cosh c=\cosh a_{1} \cosh a_{2} .
$$

Do the same for $Q^{\prime}$, obtaining triangles of side length $b_{1}, b_{2}, d$ and $b_{3}, b_{4}, d$. Now consider a parameterized family of right triangles on $[0,1]$ with adjacent sides $s a_{1}+(1-s) b_{1}$ and $s a_{2}+(1-s) b_{2}$ and opposite side $c(s)$. Differentiating (3.4) we get

$$
\sinh c \frac{d c}{d s}=\sinh a_{1} \frac{d a_{1}}{d s} \cosh a_{2}+\cosh a_{1} \sinh a_{2} \frac{d a_{2}}{d s} .
$$

Since $c$ is comparable to $a_{1}$ and $a_{2}$ we get that $\frac{d c}{d a}=O\left(\epsilon a_{1}\right)$. The argument shows $c / d=1+O(\epsilon)$. Thus we can apply Lemma 3.1 once we know the angles of the triangles are bounded below uniformly.

However, one angle of the $a_{1}, a_{2}, c$ triangle is $\pi / 2$ and the side lengths are comparable. Thus by (2.1) all three angles are comparable (and hence are uniformly bounded below). A similar argument holds for the $a_{3}, a_{4}, c$ triangle since one angle (the one between $a_{3}$ and $a_{4}$ ) is assumed to be bigger than $\theta$ and less than $\pi-\theta$.

Corollary 3.3. Suppose $H$ is a hyperbolic hexagon such that all the edge lengths $a_{1}, \ldots, a_{6}$ (in clockwise direction) are $\leq B$ and are comparable with constant $B$. Also assume three alternating angles are $\pi / 2$ and the remaining angles are bounded below by $\theta>0$ and above by $\pi-\theta$. Then there is a $C=C(\theta, B)$ so that the following holds. If $H^{\prime}$ is another such hexagon with edge lengths $b_{1}, \ldots, b_{6}$ and $\epsilon=\max _{i}\left|\log a_{i} / b_{i}\right| \leq 2$, then there is a $1+C \epsilon$ quasiconformal map from $H$ to $H^{\prime}$ which is affine on each of the edges of $H$. 
Proof. Triangulate $H$ by adding edges $\left[v_{2} v_{4}\right],\left[v_{4} v_{6}\right]$ and $\left[v_{6} v_{2}\right]$, as in Figure 6.

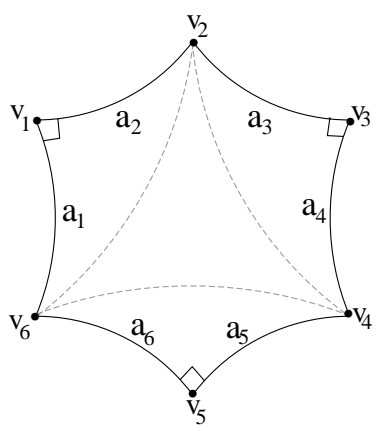

FiguRE 6

As in the previous corollary the new edges have length comparable to the edges of $H$ and if the edges of $H$ change by a factor of $1+\epsilon$ then the lengths of the new edges change by at most $1+O(\epsilon)$. Each of the three triangles which share edges with $H$ has a right angle, and hence all their angles are bounded away from zero (since their edges are all comparable).

Thus we need only show the central triangle has angles bounded away from zero. However, all of its angles are smaller than $\pi-\theta$ (since this is true for the hexagon) and since the sides are comparable, so are the angles. Finally, not all three angles can be close to 0 since this would imply the triangle has large diameter (which is impossible since it is contained in $H$ which has diameter bounded by $6 B$ ).

\section{Quasiconformal mappings of Lambert quadrilaterals}

In this section we use the result about triangles from the previous section to prove a result about mappings of certain special quadrilaterals. In Section 6 on mappings of $Y$-pieces these quadrilaterals will correspond to neighborhoods of very short geodesic boundaries and the estimate we prove here will give the exponential decay we want.

A hyperbolic quadrilateral is called a Lambert quadrilateral if it has three right angles (after J.H. Lambert, 1728-1777, see page 156 of [2]). We denote the fourth angle $\phi$ and the four sides (going clockwise, starting at $\phi$ ) will be labeled $b, x, a, y$ as in Figure 7 .

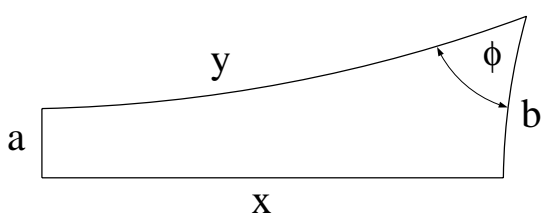

Figure 7: A Lambert quadrilateral 
Given such a quadrilateral $Q$, we will sometimes let the letter $a$ denote the edge of $Q$ and sometimes let it denote the length of the edge. The usage will generally be clear from context (e.g. it is a length in $\sinh a$ but the side itself in $\rho(z, a))$, but we will write " $a$-side" when necessary. Similarly for the other sides of $Q$.

The quadrilateral is determined by any two side lengths or one side length and the angle $\phi$, as is evident from the following two relations (Theorem 7.17.1 of [2])

$$
\begin{aligned}
& \sinh a \sinh x=\cos \phi \\
& \cosh a=\cosh b \sin \phi
\end{aligned}
$$

We claim these imply

$$
\cosh b \sinh x=\sinh y \text {. }
$$

To prove this use (4.1) and (4.2) to get

$$
\begin{aligned}
\sin \phi \cosh b & =\cosh a=\left(1+\sinh ^{2} a\right)^{1 / 2} \\
& =\left(1+\frac{\cos ^{2} \phi}{\sinh ^{2} x}\right)^{1 / 2}=\left(\frac{\cosh ^{2} x-1+\cos ^{2} \phi}{\sinh ^{2} x}\right)^{1 / 2} \\
& =\left(\frac{\cosh ^{2} y \sin ^{2} \phi-\sin ^{2} \phi}{\sinh ^{2} x}\right)^{1 / 2}=\frac{\sin \phi \sinh y}{\sinh x},
\end{aligned}
$$

which proves (4.3). We will think of $b$ as fixed, but allow $a$ to vary and consider $\phi, x$ and $y$ as functions of $a$.

Lemma 4.1. Suppose $Q$ is a Lambert quadrilateral, labeled as above. If $b$ is fixed and $a \leq b / 2$ is allowed to vary then

$$
\begin{gathered}
\frac{b}{a} \simeq \sinh x \\
\frac{d \phi}{d a}=O(a) \\
\frac{d x}{d a}=\frac{-1}{\sinh a}+O(a) \\
\frac{d y}{d a}=\frac{-1}{\sinh a}+O(a)
\end{gathered}
$$

The constants depend only on an upper bound for $b$. 
Proof. We recall an estimate which we will use frequently. Note that if $x>0$,

$$
1+e^{-2 x} \leq \frac{\cosh x}{\sinh x} \leq \frac{1+e^{-2 x}}{1-e^{-2 x}}
$$

and hence $(\cosh x) /(\sinh x)=1+O\left(e^{-2 x}\right)$ with a constant that depends only on a lower bound for $x$.

First we prove (4.4). By (4.1) and (4.2),

$$
\begin{aligned}
\sinh ^{2} x & =\frac{\cos ^{2} \phi}{\sinh ^{2} a}=\frac{1-\sin ^{2} \phi}{\sinh ^{2} a} \\
& =\frac{\cosh ^{2} b-\cosh ^{2} a}{\sinh ^{2} a \cosh ^{2} b} \\
& \simeq \frac{b^{2}}{a^{2}},
\end{aligned}
$$

where we have used $a \leq b / 2$ to deduce $\frac{1}{C} b^{2} \leq \cosh ^{2} b-\cosh ^{2} a \leq C b^{2}$.

Next we prove (4.5). By (4.2)

$$
\sin \phi=\frac{\cosh a}{\cosh b} \leq \frac{\cosh b / 2}{\cosh b}<1,
$$

with a bound depending only on $b$. Differentiating the equality gives

$$
\frac{d \phi}{d a}=\frac{\sinh a}{\cos \phi \cosh b}=O(a) \text {. }
$$

Also note for later use that if $t<s \leq b / 2$ then

$$
\phi(s)-\phi(t)=\int_{s}^{t} O(a) d a=O\left(\left|s^{2}-t^{2}\right|\right) .
$$

To prove (4.6), note that by (4.1) we have

$$
\sinh x=\frac{\cos \phi}{\sinh a}
$$

and so differentiating and using (4.1) and (4.10),

$$
\begin{aligned}
\frac{d x}{d a} & =-\frac{1}{\cosh x} \frac{-\sin \phi \frac{d \phi}{d a} \sinh a-\cos \phi \cosh a}{\sinh ^{2} a} \\
& =-\frac{1}{\cosh x} \frac{-\sin \phi \sinh ^{2} a /(\cos \phi \cosh b)-\sinh a \sinh x \cosh a}{\sinh ^{2} a} \\
& =-\frac{1}{\sinh a}\left[\frac{-\sin \phi \sinh a}{\cos \phi \cosh b \cosh x}+\cosh a \frac{\sinh x}{\cosh x}\right] \\
& =\frac{-1}{\sinh a}\left[O\left(a^{2}\right)+\left(1+O\left(a^{2}\right)\right)\left(1+O\left(e^{-2 x}\right)\right)\right] \\
& =\frac{-1}{\sinh a}\left(1+O\left(a^{2}\right)\right),
\end{aligned}
$$


where the fourth equality uses the fact (4.9) that $\cos \phi$ is bounded away from zero (depending only on $b$ ), that $\cosh x \simeq 1 / a$ and the fact that (4.4) implies

$$
\frac{1}{C} a \leq e^{-x} \leq C a
$$

if $a \leq b / 2$ (for some $C$ depending on $b$ ). Also note that

$$
\left|\frac{1}{a}-\frac{1}{\sinh a}\right|=O(a) .
$$

Integrating we get

$$
|x(s)-x(t)|=\int_{s}^{t} \frac{1}{a}+O(a) d a=\left|\log \frac{t}{s}\right|+O\left(\left|s^{2}-t^{2}\right|\right) .
$$

Finally, we prove (4.7). If we differentiate (4.3) with respect to $x$ we get

$$
\cosh b \cosh x=\cosh y \frac{d y}{d x} .
$$

Now divide by $\cosh y$ and use (4.2) to get

$$
\frac{d y}{d x}=\cosh a=1+O\left(a^{2}\right)
$$

The chain rule then gives

$$
\frac{d y}{d a}=\frac{d y}{d x} \frac{d x}{d a}=\frac{-1}{\sinh a}\left(1+O\left(a^{2}\right)\right)\left(1+O\left(a^{2}\right)\right)=\frac{-1}{\sinh a}+O(a),
$$

as desired.

Next we wish to prove the main estimate of this section:

Lemma 4.2. Assume $b<1 / 2$. Suppose $Q_{s}, Q_{t}$ are Lambert quadrilaterals with a-sides of length $s$ and $t$ respectively and have equal length opposite sides $b$ in both cases. Assume $s / 10 \leq t \leq s \leq b / 2$. Let $\epsilon=(s-t) / s$. There is a $C<\infty$ depending only on $b$ so that the following holds. There is a quasiconformal mapping $f: Q_{s} \rightarrow Q_{t}$ which is affine on the edges $a$ and $b$ and whose dilatation satisfies

$$
K(x) \leq\left\{\begin{array}{ll}
1+C \epsilon & \rho(x, a) \leq 1 \\
1+C \epsilon \exp (-2 \rho(x, a)), & \rho(x, a) \geq 1 .
\end{array} .\right.
$$


Proof. We know from (4.4) that $x=x(s)$ is bounded away from zero uniformly (in terms of $b$ ). If $x \leq 1$, then the lemma follows from Corollary 3.2. Thus we may assume $x>1$.

Choose points $x_{0}, \ldots x_{n}$ along the $x$-side of $Q_{s}$ inductively as follows. The point $x_{0}$ is the corner of $Q_{s}$ where the $x$ and $b$ sides meet. The point $y_{0}$ is the other endpoint of the $b$-side. The point $x_{1}$ is the point on the $x$-side which is distance $b$ from $x_{0}$. Consider the geodesic segment which is perpendicular to the $x$-side at $x_{1}$ and let $y_{1}$ be the point where it hits the $y$-side of $Q_{s}$ and let $d_{1}$ be its length. Let $x_{2}$ be the point on the $x$-side of $Q_{s}$ which is distance $d_{1}$ from $x_{1}$ (and so that $x_{1}$ separates $x_{2}$ and $x_{0}$ ). In general, given $x_{n-1}$, consider the geodesic segment perpendicular to $x$ through $x_{n-1}$, let $y_{n-1}$ be the point of intersection of this geodesic with the $y$-side of $Q_{s}$ and let $d_{n-1}$ be the distance from $x_{n-1}$ and $y_{n-1}$. Then let $x_{n}$ be the point on the $x$-side of $Q_{s}$ which is distance $d_{n-1}$ from $x_{n-1}$ and which is separated from $x_{0}$ by $x_{n-1}$. See Figure 8 .

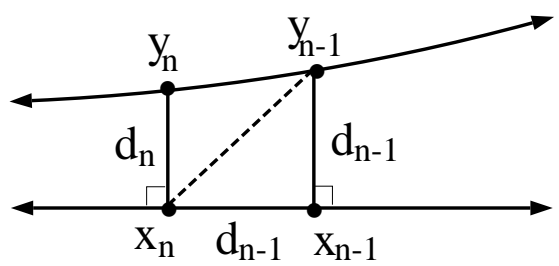

Figure 8: Definition of $S_{n-1}$

Continue until the first time that the distance from $x_{n}$ to the $a$-side of $Q_{s}$ is $<1$. Let $N$ denote the index when this occurs. For $n=1, \ldots N$, let $S_{n} \subset Q_{s}$ denote the quadrilateral with corners $x_{n-1}, x_{n}, y_{n}, y_{n-1}$. Let $T=Q_{s} \backslash \cup_{1}^{N} Q_{n}$. See Figure 9.

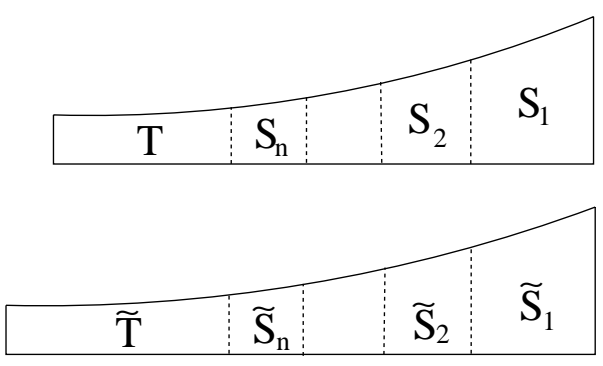

Figure 9: Decomposing $Q_{s}$ and $Q_{t}$

Now divide the quadrilateral $Q_{t}$ in a similar way. Choose points $\tilde{x}_{n}$ on its $x$ side so that $\tilde{x}_{0}$ is the corner of its $b$ and $x$ sides and $\rho\left(\tilde{x}_{n}, \tilde{x}_{0}\right)=\rho\left(x_{n}, x_{0}\right)$ for $n=1, \ldots N$. Take the perpendiculars to the $x$-side of $Q_{t}$ though these points and let $\tilde{y}_{n}$ be the points of intersection with the $y$-side of $Q_{t}$ and $\tilde{d}$ be their lengths. Let $\tilde{S}_{n}$ be the corresponding quadrilaterals and let $\tilde{T}=Q_{t} \backslash \cup_{1}^{N} \tilde{S}_{n}$. See Figure 9. 
By Lemma 4.1 we know $|\phi(s)-\phi(t)| \leq C\left(s^{2}-t^{2}\right)=O(s(s-t))$. The segment $d_{n}$ meets the $y$ edges of $Q_{s}$ and $Q_{t}$ at angles between $\phi$ and $\pi / 2$. Thus (4.1), (4.12) and (4.4) imply

$$
d_{n}-\tilde{d}_{n} \leq C s(s-t) e^{\rho\left(x_{n}, x_{0}\right)}=C\left(1-\frac{t}{s}\right) e^{-2 x} e^{\rho\left(x_{n}, x_{0}\right)}=C \epsilon e^{-x-\rho\left(x_{n}, a\right)} .
$$

See Figure 10 (in this figure, the vertex of $\phi$ is at the origin and the $b$-sides for the two quadrilaterals coincide).

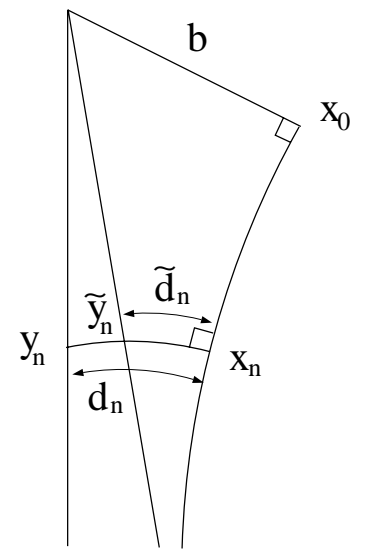

FIGURE 10: Estimating $d_{n}-\tilde{d}_{n}$

Using (4.4) and the fact that $d_{n}$ is the $b$-side of a Lambert quadrilateral itself, we see

$$
d_{n} \geq C a \sinh \rho\left(x_{n}, a\right) \geq C e^{-x} e^{\rho\left(x_{n}, a\right)}=C e^{-\rho\left(x_{n}, x_{0}\right)} .
$$

Thus

$$
d_{n} / \tilde{d}_{n}=1+O\left(\epsilon e^{-2 \rho\left(x_{n}, a\right)}\right) .
$$

Thus by Corollary 3.2 there is a $1+O\left(\epsilon e^{-2 \rho\left(x_{n}, a\right)}\right)$-quasiconformal map from $S_{n}$ to $\tilde{S}_{n}$. This gives the lemma on the $S_{n}$ 's.

Subdivide $T$ (which by our choice of $N$ has $x$-side of length between $1 / 2$ and 1) into subquadrilaterials with equal length $x$-sides $\simeq a$. The corresponding side of $\tilde{T}$ is only longer by a factor of $\log \frac{s}{t}+O(a(s-t))=O(s-t)$ by (4.13) and so we can apply Corollary 3.2 and get maps on each subquadrilateral with quasiconformal constant $1+O(\epsilon)$.

The estimate in the previous lemma for the case $\rho(x, a) \geq 1$ did not use the hypothesis that $t \geq s / 10$, so that estimate is true in general. For $t \leq s / 10$ the estimate for $\rho(x, a)<1$ becomes $K(x) \leq C s / t$. To prove this, note that because $T$ and $\tilde{T}$ are both Lambert quadrilateral themselves, we may place them in the hyperbolic upper half-plane so that the $a$-sides lie on 
the imaginary axis, the $x$-side lies on the circle $|z|=1$ and the $y$-sides line on circles of the form $|z|=r$ for $r=e^{s}$ and $r=e^{t}$. The remaining $b$ sides lie on circles centered on the real axis. See Figure 11. Apply logarithims to conformally map to horizontal strips.

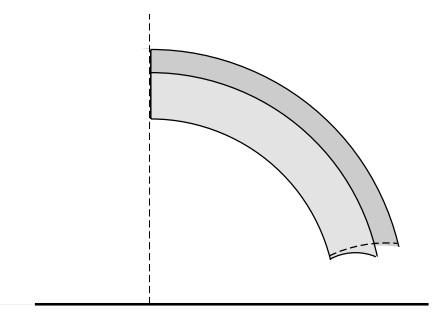

Figure 11: $T$ and $\tilde{T}$

We now map $T$ to $\tilde{T}$ in a series of steps as illustrated in Figure 12.

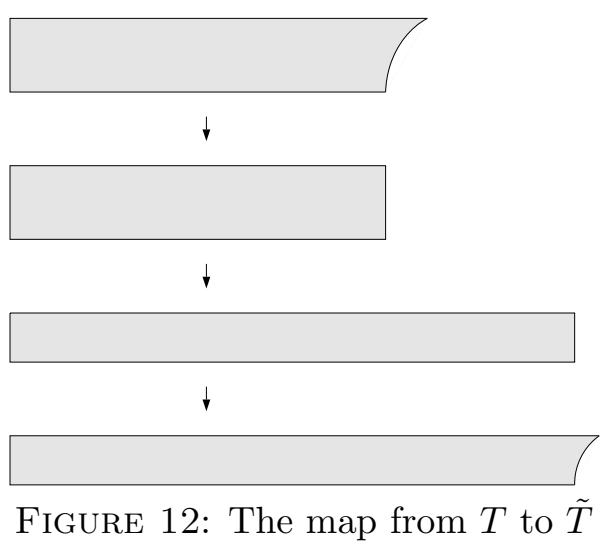

First we map $T$ to $W_{1} \subset P_{1}$ with the $b$ side going to a radial line segment and hyperbolic arclength on this edge being mapped affinely onto arclength of the image. This can be done with quasiconformal constant $C_{1}$ bounded only depending on $b$. Next we map $W_{1} \rightarrow W_{2} \subset \tilde{T}$ by the map $r e^{i \theta} \rightarrow$ $r^{s / t} e^{i\left(\frac{p}{i} 2-a\left(\frac{\pi}{2}-\theta\right)\right)}$ where $a$ is chosen so that $\partial W_{1} \cap\{|z|=1\}$ is mapped onto the $a_{2}$ edge of $P_{\lambda}$. Since this edge of $W_{1}$ has (Euclidean) length uniformly bounded below, and the (Euclidean) length of the image is at most $\pi / 2$, we see that $a$ is uniformly bounded. Thus this map has quasiconformal constant $\leq C_{2} \frac{s}{t}$, where $C_{2}$ is absolute. Finally we map $W_{2}$ to $P_{\lambda}$ by mapping the radial edge onto the $b$ edge of $\tilde{T}$ with arclength mapping affinely. As above, this can be done with constant $C_{1}$ which depends only on $b$. Composing these steps gives the desired map with a quasiconformal constant of $C_{1}^{2} C_{2} \frac{s}{t}$. This finishes the proof of the estimate.

If $t>s$, then it is easy to formulate and prove the corresponding estimates by considering the inverse of the map constructed above. 


\section{Decomposing a $L$-bounded hexagon}

Let $H$ be a hyperbolic hexagon with all right angles and suppose the sides have lengths $a_{1}, b_{3}, a_{2}, b_{1}, a_{3}, b_{2}$, ordered counter-clockwise around the boundary (so $a_{j}$ is opposite $b_{j}$ ). See Figure 13 .

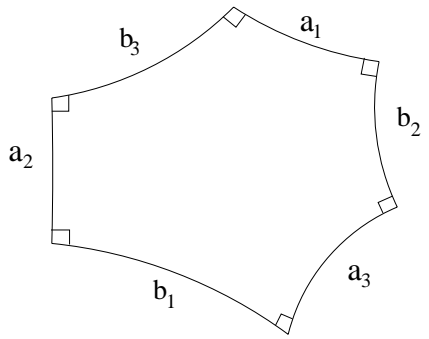

FiguRE 13: Labeling sides of a hexagon

The following two relations are Theorems 7.19.1 and 7.19.2 of [2],

$$
\begin{gathered}
\frac{\sinh a_{1}}{\sinh b_{1}}=\frac{\sinh a_{2}}{\sinh b_{2}}=\frac{\sinh a_{3}}{\sinh b_{3}}, \\
\cosh b_{1} \sinh a_{2} \sinh a_{3}=\cosh a_{1}+\cosh a_{2} \cosh a_{3} .
\end{gathered}
$$

Note that this easily implies that right hexagon is determined by the lengths of three alternating sides.

Throughout this section we will assume that the three sides $a_{1}, a_{2}, a_{3}$ are all bounded by some $L<\infty$. Such a hexagon will be called $L$-bounded. However, if any of these sides is very short then some of the remaining $b$ edges will be long. In this section we want to show that any $L$-bounded hexagon $H$ can be divided into a central hexagon $H_{0}$, all of whose edges are bounded and bounded away from zero in terms of $L$, and at most three Lambert quadrilaterals.

To accomplish this, suppose we have two numbers $a, b$ (to be chosen later) with $a \leq \frac{1}{2} b<1 / 4$. If $a_{j} \leq a, j=1,2,3$, then form a Lambert quadrilateral, $Q_{j}$, with $a_{j}$ as one side, two other sides lying along the edges $b_{j-1}$ and $b_{j+1}$ (here and below indices are taken mod 3 in $\{1,2,3\}$ ) and the fourth edge has length $b$ and makes a right angle with $b_{j-1}$. See Figure 14 .

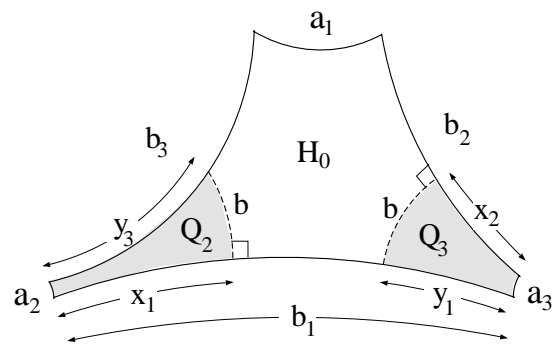

Figure 14: A hexagon with two short sides 
The unique non-right angle in $Q_{j}$ will be denotes $\phi_{j}$. Let $x_{j-1}$ be the length of the edge of $Q_{j}$ which lies in $b_{j-1}$ and let $y_{j+1}$ be the length of the edge of $Q_{j}$ which lies on $b_{j+1}$. We shall see below that $Q_{j}$ is a subset of $H$ if $a$ and $b$ are chosen correctly. If $a_{j}>a$ then we set $Q_{j}=\emptyset$ and $x_{j}=y_{j}=0$. Let $H_{0}=H \backslash \cup_{j=1}^{3} Q_{j}$. The six sides of $H_{0}$ are in an obvious correspondence with those of $H$ and will say "the side of $H_{0}$ in position $a_{j}$ (or $\left.b_{j}\right)$ ".

Lemma 5.1. There is a choice of $a, b$ so that $H_{0} \subset H$ and the side lengths of $H_{0}$ are bounded and bounded away from zero depending only on $L, a, b$.

Proof. Since the sides of $H_{0}$ in positions $a_{1}, a_{2}, a_{3}$ are all $\leq L$ and by construction have length $\geq a$, we only have to check the sides in positions $b_{1}, b_{2}, b_{3}$. Without loss of generality it suffices to consider just $b_{1}$. There are four cases depending on whether $a_{2}>a$ and $a_{3}>a$.

Case 1: First suppose both $a_{2}, a_{3}>a$, so that the $b_{1}$-edge of $H_{0}$ is the same as the corresponding edge of $H$, i.e., has length $b_{1}$. By (5.2),

$$
\cosh b_{1}=\frac{\cosh a_{1}+\cosh a_{2} \cosh a_{3}}{\sinh a_{2} \sinh a_{3}} \leq \frac{2 e^{L}+2 e^{2 L}}{a^{2}}
$$

and so $b_{1}$ is bounded above depending only on $a$ and $L$. On the other hand, by (5.2) and (4.8)

$$
\begin{aligned}
\cosh b_{1}=\frac{\cosh a_{1}+\cosh a_{2} \cosh a_{3}}{\sinh a_{2} \sinh a_{3}} & \geq e^{-2 L}+\left(1+e^{-2 a_{2}}\right)\left(1+e^{-2 a_{3}}\right) \\
& \geq 1+2 e^{-2 a}
\end{aligned}
$$

which implies $b_{1}$ is bounded below depending only on $a$.

Case 2: Next suppose $a_{2} \leq a$ and $a_{3}>a$. Then the side of $H_{0}$ in position $b_{1}$ has length $b_{1}-x_{1}$. By $(4.1)$

$$
\sinh x_{1}=\frac{\cos \phi_{2}}{\sinh a_{2}}
$$

and by (5.2),

$$
\begin{aligned}
\cosh b_{1} & =\frac{1}{\sinh a_{2}} \frac{\cosh a_{1}+\cosh a_{2} \cosh a_{3}}{\sinh a_{3}} \\
& \geq \frac{1}{\sinh a_{2}} \frac{\cosh a_{3}}{\sinh a_{3}} \\
& \geq \frac{1}{\sinh a_{2}} .
\end{aligned}
$$

This implies

$$
2 e^{b_{1}} \geq e^{b_{1}}+e^{-b_{1}}=2 \cosh b_{1} \geq \frac{2}{\sinh a_{2}} \geq \frac{2 C}{a_{2}}
$$


for some $C=C(b)$ since $a_{2} \leq a<b$. Hence

$$
b_{1} \geq \log \frac{1}{a_{2}}+\log C(b) \geq \log \frac{1}{b}+\log C(b),
$$

independent of $a$ or $L$. By (4.2)

$$
\sin \phi_{2}=\frac{\cosh a_{2}}{\cosh b} \geq \frac{1}{\cosh b},
$$

so if $b$ is small enough then $\phi_{2}$ is a close as we wish to $\pi / 2$ (independent of a). Assume we have fixed $b$ so small that $\cos \phi_{2} \leq \frac{1}{2}$ and $b_{1} \geq 1$ (using (5.4). By (4.1) and (5.3)

$$
\sinh x_{1}=\frac{\cos \phi_{2}}{\sinh a_{2}} \leq \frac{1 / 2}{\sinh a_{2}} \leq \frac{1}{2} \cosh b_{1},
$$

and since $b_{1} \geq 1$ this implies $b_{1}-x_{1} \geq C_{1}$ for some absolute $C_{1}$ which does not depend on $a, b$ or $L$ (as long as $b$ satisfies the choices made above).

To prove the upper bound, note that if $a_{1}, a_{2} \leq L$ and $a_{3} \geq a$, then

$$
\begin{aligned}
\cosh b_{1} & =\frac{1}{\sinh a_{2}} \frac{\cosh a_{1}+\cosh a_{2} \cosh a_{3}}{\sinh a_{3}} \\
& \leq \frac{1}{\sinh a_{2}}\left(\frac{\cosh a_{1}}{\sinh a_{3}}+\cosh a_{2} \frac{\cosh a_{3}}{\sinh a_{3}}\right) \\
& \leq \frac{1}{\sinh a_{2}}\left(\frac{e^{L}}{a}+e^{L}\left(1+e^{-2 a}\right)\right) \\
& \leq \frac{A(a, L)}{\sinh a_{2}}
\end{aligned}
$$

Thus

$$
\sinh x_{1}=\frac{\cos \phi_{2}}{\sinh a_{2}} \geq \frac{\cos \phi_{2}}{A(a, L)} \cosh b_{1},
$$

which implies $b_{1}-x_{1} \leq C_{2}$ for some absolute $C_{2}$ which depends on $a$ and $L$. This proves that $b_{1}-x_{1}$ is bounded above and below in terms of $a, b$ and $L$.

Case 3: Next assume $a_{2}>a$, but $a_{3} \leq a$. Since $a_{2}$ and $a_{3}$ play symmetric roles in (5.2), the argument above shows that $b_{1}-x_{2}$ is bounded above and below uniformly. We shall now show that $x_{2}-y_{1}$ is small, and hence that $b_{1}-y_{1}$ is also bounded above and below. By (4.2)

$$
\cosh y_{1} \sin \phi_{3}=\cosh x_{2} \text {. }
$$

Since $\sin \phi_{3}<1$, this implies

$$
y_{1}>x_{2} \text {. }
$$


Thus $b_{1}-y_{1}$ is bounded above since $b_{1}-x_{2}$ is. On the other hand,

$$
\sin \phi_{3}=\frac{\cosh x_{2}}{\cosh y_{1}}=\frac{e^{x_{2}}+e^{-x_{2}}}{e^{y_{1}}+e^{-y_{1}}}=e^{x_{2}-y_{1}} \frac{1+e^{-2 x_{2}}}{1+e^{-2 y_{1}}},
$$

and hence

$$
\begin{aligned}
y_{1} & =x_{2}+\log \frac{1}{\sin \phi_{3}}+\log \left(1+e^{-2 x_{2}}\right)-\log \left(1+e^{-2 y_{1}}\right) \\
& \leq x_{2}+\log \frac{1}{\sin \phi_{3}}+\log \left(1+e^{-2 x_{2}}\right) \\
& \leq x_{2}+C
\end{aligned}
$$

where $C$ may be as small as we wish if $b$ is small enough. Taking it to be less than $\frac{1}{2} C_{1}$ (which did not depend on $b$ ) we get

$$
b_{1}-y_{1} \geq \frac{1}{2} C_{1}
$$

as our lower bound if $b$ is small enough (independent of $L$ ).

Note that the proof of the previous two cases shows that $x_{j} \leq b_{j}$ and $y_{j} \leq b_{j}$ and hence the quadrilaterals $Q_{j}$ are really subsets of $H$, as claimed in the lemma.

Case 4: Finally assume both $a_{2}, a_{3} \leq a$. By (5.2),

$$
\cosh b_{1}=\frac{\cosh a_{1}+\cosh a_{1} \cosh a_{3}}{\sinh a_{2} \sinh a_{3}} \geq \frac{2}{\sinh a_{2} \sinh a_{3}},
$$

whereas by the middle of (5.5),

$$
\begin{aligned}
& \sinh x_{1} \leq \frac{1}{2} \frac{1}{\sinh a_{2}}, \\
& \sinh x_{2} \leq \frac{1}{2} \frac{1}{\sinh a_{3}} .
\end{aligned}
$$

Thus

$$
\sinh x_{1} \sinh x_{2} \leq \frac{1}{8} \cosh b_{1},
$$

and because $b_{1} \geq 1$ this implies $b_{1}-\left(x_{1}+x_{2}\right) \geq C_{3}$, with $C_{3}$ independent of $b$. Choose $b$ so small that $\left|x_{2}-y_{1}\right| \leq C_{3} / 2$ and this gives the desired lower bound.

On the other hand, by (5.2),

$$
\cosh b_{1}=\frac{\cosh a_{1}+\cosh a_{2} \cosh a_{3}}{\sinh a_{2} \sinh a_{3}} \leq \frac{e^{L}+e^{2 a}}{\sinh a_{2} \sinh a_{3}}
$$


which implies by (5.5),

$$
\sinh x_{1} \sinh x_{2} \geq \frac{1}{A(L, a, b)} \cosh b_{1}
$$

which implies $b_{1}-\left(x_{1}+x_{2}\right)$ is bounded above, depending only on $L, a, b$. Since $y_{1} \geq x_{2}$, this gives the desired upper bound.

Note that this shows the quadrilaterals $Q_{1}, Q_{2}$ and $Q_{3}$ are disjoint. This completes all the cases and hence finishes the proof of the lemma.

Next we record a calculation which we will need later. We will think of the $a_{1}$ and $a_{3}$ sides of our right hexagon as having fixed length, but as $a_{2}$ as varying and we wish to compute the rate of change of the $b$-sides. Since $b_{1}$ and $b_{3}$ play symmetric roles we need only compute the derivatives for $b_{1}$ and $b_{2}$.

Lemma 5.2. With notation as above

$$
\begin{aligned}
\frac{d b_{1}}{d a_{2}} & =\frac{-1}{\sinh a_{2}}\left(1+O\left(e^{-2 b_{3}}\right)\right), \\
\frac{d b_{2}}{d a_{2}} & =O\left(a_{2}\right),
\end{aligned}
$$

where the constants depend only on $L$, the upper bound for $a_{1}, a_{2}, a_{3}$.

Proof. If we think of (5.2) as giving $b_{1}$ as a function of $a_{2}$ with $a_{1}$ and $a_{3}$ fixed, and differentiate, we get

$$
\begin{aligned}
\frac{d b_{1}}{d a_{2}} & =\frac{\sinh ^{2} a_{2} \cosh a_{3}-\left(\cosh a_{1}+\cosh a_{2} \cosh a_{3}\right) \cosh a_{2}}{\sinh b_{1} \sinh ^{2} a_{2} \sinh a_{3}} \\
& =\frac{1}{\sinh a_{2}} \frac{\sinh a_{2}^{2} \cosh a_{3}-\cosh a_{1} \cosh a_{2}-\cosh ^{2} a_{2} \cosh a_{3}}{\sinh b_{1} \sinh a_{2} \sinh a_{3}} \\
& =\frac{1}{\sinh a_{2}} \frac{\left(\sinh a_{2}^{2}-\cosh a_{2}^{2}\right) \cosh a_{3}-\cosh a_{1} \cosh a_{2}}{\sinh b_{1} \sinh a_{2} \sinh a_{3}} \\
& =\frac{-\cosh a_{3}-\cosh a_{1} \cosh a_{2}}{\sinh a_{2} \sinh b_{1} \sinh a_{2} \sinh a_{3}} \\
& =\frac{-\cosh b_{3} \sinh a_{1} \sinh a_{2}}{\sinh a_{2} \sinh b_{1} \sinh a_{2} \sinh a_{3}} \\
& =\frac{-\cosh b_{3} \sinh a_{1}}{\sinh a_{2} \sinh b_{1} \sinh a_{3}} \\
& =\frac{-\cosh b_{3} \sinh a_{3}}{\sinh a_{2} \sinh b_{3} \sinh a_{3}} \\
& =\frac{-\cosh b_{3}}{\sinh a_{2} \sinh b_{3}} \\
& =\frac{-1}{\sinh a_{2}}\left(1+O\left(e^{-2 b_{3}}\right)\right)
\end{aligned}
$$


where the fifth equality holds by (5.2) and the seventh holds by (5.1) and the constant in the last line only depends on a lower bound for $b_{3}$. We know by (5.2) that

$$
\begin{aligned}
\cosh b_{3} & =\frac{\cosh a_{1}+\cosh a_{2} \cosh a_{3}}{\sinh a_{2} \sinh a_{3}} \\
& \geq \frac{1}{\sinh a_{2} \sinh a_{3}}+\left(\frac{\cosh a_{2}}{\sinh a_{2}} \frac{\cosh a_{3}}{\sinh a_{3}}\right) \\
& \geq e^{-2 L}+1,
\end{aligned}
$$

since $a_{2}, a_{3} \leq L$. Thus $b_{3}$ is bounded away from zero depending only on $L$ and so our estimate of the derivative above depends only on $L$.

To see what happens to the side corresponding to $b_{2}$ think of (5.2) as giving $b_{2}$ as a function of $a_{2}$ with $a_{1}, a_{3}$ fixed. Differentiating, we get

$$
\frac{d b_{2}}{d a_{2}}=\frac{\sinh a_{2}}{\sinh b_{2} \sinh a_{1} \sinh a_{3}} .
$$

By Theorem 17.18.1 of [2],

$$
\cosh t=\sinh b_{2} \sinh a_{3},
$$

where $t$ is the length of the common orthogonal between sides $a_{1}$ and $b_{1}$. See Figure 15.

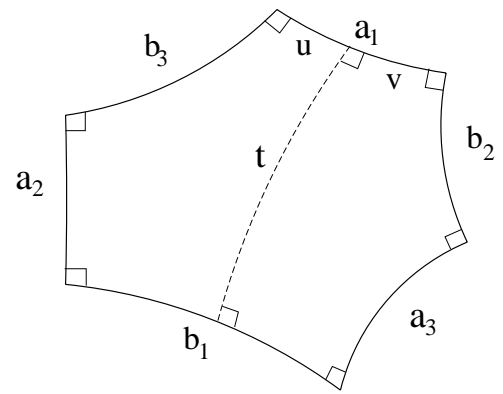

Figure 15: Definition of $t$

Let $u+v=a_{1}$ be the lengths of the subintervals of $a_{1}$ on either side of $t$ as in Figure 15. On page 161 of [2] it is shown that

$$
\sinh a_{1} \sinh t \geq \sinh u \sinh t=\cosh a_{2} \geq 1,
$$

and hence

$$
\frac{d b_{2}}{d a_{2}}=\frac{\sinh a_{2}}{\cosh t \sinh a_{1}} \leq \sinh a_{2}=O\left(a_{2}\right)
$$


Lemma 5.3. Given $L<\infty$, there is a $C=C(L)<\infty$ so that the following holds. Suppose $H_{1}$ is right, hyperbolic hexagon and alternate side lengths $a_{1}, a_{2}=s, a_{3}$ and suppose $s \leq L$. Suppose $H_{2}$ is another right hexagon with alternate side lengths $a_{1}, a_{2}=t, a_{3}$. Assume $s$ and $t$ are both less than $a$. Then the central hexagons $H_{0,1} H_{0,2}$ of $H_{1}$ and $H_{2}$ as described above have corresponding sides and angles which differ by less than $C\left|s^{2}-t^{2}\right|$.

Proof. Five of the angles in $H_{0,1}$ agree exactly with the corresponding angles in $H_{0,2}$. The only one that may disagree is the angle between the sides in the $a_{2}$ and $b_{3}$ positions. This angle is complementary to $\phi_{2}$. So by (4.11) this angle in the two hexagons differs by less than $O\left(\left|s^{2}-t^{2}\right|\right)$ as desired.

The sides of $H_{0,1}$ and $H_{0,2}$ which correspond to positions $a_{1}, a_{2}, a_{3}$ are the same length. Thus we need only consider the other three sides $b_{1}, b_{2}, b_{3}$ (and since $b_{1}$ and $b_{3}$ play symmetric roles, it is enough to consider only $b_{1}$ and $b_{2}$ ). By Lemma 5.2, $\frac{d b_{2}}{d a_{2}}=O\left(a_{2}\right)$. Moreover, the side lengths of the quadrilaterals $Q_{1}$ and $Q_{3}$ (if they occur) don't depend on $a_{2}$ and hence, integrating as in (4.11), we see that the side lengths of the central hexagons for $H_{1}$ and $H_{2}$ in position $b_{2}$ differ by at most $O\left(\left|s^{2}-t^{2}\right|\right)$.

Next we consider the sides in position $b_{1}$. By the proof of Lemma 5.1 we know $b_{3} \geq y_{3} \geq x_{1}$. Thus by (4.12) we get

$$
e^{-2 b_{3}} \leq e^{-2 x_{1}} \leq a_{2}^{2}
$$

Hence, from (4.6) and (5.9) we deduce

$$
\frac{d x_{1}}{d a_{2}}-\frac{d b_{1}}{d a_{2}}=\frac{-1}{\sinh a_{2}}\left(1+O\left(a_{2}^{2}\right)\right)-\frac{-1}{\sinh a_{2}}\left(1+O\left(a_{2}^{2}\right)\right)=O\left(a_{2}\right)
$$

Since $y_{1}$ does not depend on $a_{2}$, this implies the sides of the central hexagons of $H_{1}$ and $H_{2}$ corresponding to $b_{1}$ differ by $O\left(\left|s^{2}-t^{2}\right|\right)$.

Finally we have to consider the sides in position $b_{3}$. By (4.7),

$$
\frac{d y_{3}}{d a_{2}}=\frac{d y_{3}}{d x_{1}} \frac{d x_{1}}{d a_{2}}=\frac{1}{\sinh a_{2}}\left(1+O\left(a_{2}^{2}\right)\right)
$$

with constant depending only on $b$. Since the roles of $b_{1}$ and $b_{3}$ are symmetric, we also have

$$
\frac{d b_{3}}{d a_{2}}=\frac{1}{\sinh a_{2}}\left(1-O\left(a_{2}^{2}\right)\right)
$$

and so we can complete the proof just as in the previous case. 
Lemma 5.4. Suppose $H_{1}$ and $H_{2}$ are two L-bounded right hexagons with a-sides $\left(a_{1}^{1}, a_{2}^{1}, a_{3}^{1}\right)$ and $\left(a_{1}^{2}, a_{2}^{2}, a_{3}^{2}\right)$ respectively. Assume $a_{1}^{1}=a_{1}^{2}, a_{3}^{1}=a_{3}^{2}$ and $a_{2}^{2}=(1-\epsilon) a_{2}^{1} \leq a_{2}^{1}$. Then there is a quasiconformal map $f: H_{1} \rightarrow H_{2}$ which is affine on the a-sides, is isometric on the boundary quadrilaterals associated to $a_{1}^{1}$ and $a_{3}^{1}$ and has dilatation satisfying

$$
K_{f}(z) \leq 1+C \epsilon \exp \left(-2 \rho\left(z, a_{1}^{1}\right)\right)
$$

Proof. Since the quadrilaterals corresponding to sides $a_{1}$ and $a_{3}$ are identical for both hexagons, we may clearly take the map to be isometric in them. If $a_{1}^{1} \geq a$ (where $a$ is as in Lemma 5.1) then apply Lemma 3.3 to $H_{1} \backslash\left(Q_{1} \cup Q_{3}\right)$ using the estimates of Lemma 5.3. This gives the desired estimate since this region has bounded diameter. If $a_{1}^{1}<a$ then apply Lemma 3.3 to $H_{1} \backslash\left(Q_{1} \cup Q_{2} \cup Q_{3}\right)$ to get the estimate in the central hexagon and apply Lemma 4.2 to the quadrilateral $Q_{1}$.

\section{Quasiconformal mappings of $\mathrm{Y}$-pieces}

A Y-piece is a bordered Riemann surface which is topologically a sphere with three disks removed and in which each of the three boundary components is a hyperbolic geodesic. A generalized Y-piece is similarly defined, except that we also allow boundaries of length zero, i.e., instead of removing a disk we may remove a point.

A $Y$-piece can always be realized as a hyperbolic octagon with three side pairings. Moreover, there is a line of symmetry which divides the octagon into two isometric right hexagons. The alternate sides of these hexagons are given by $a_{1}=a / 2, a_{2}=b / 2$ and $a_{3}=c / 3$. See Figure 16 .
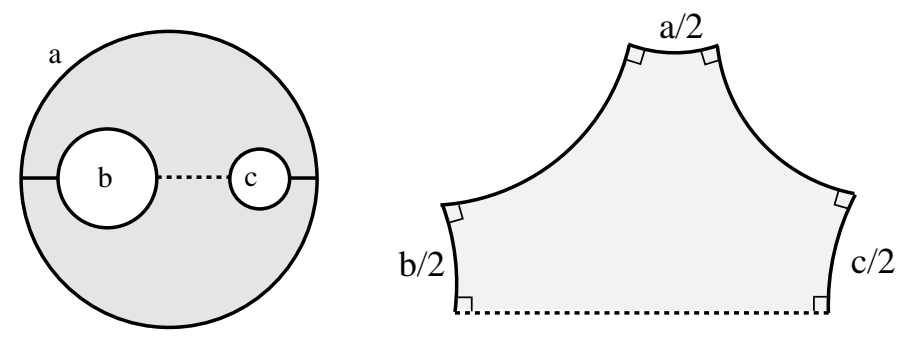

Figure 16: Parameterizing a Y-piece

As noted following (5.2), a right hexagon is determined by three alternating sides, and so we see that a $Y$ piece is uniquely determined by its three side lengths.

The part of $Y$ corresponding to the central hexagons will be called the central piece of $Y$. Note that the diameter of the central part is bounded 
depending only on $L$. The parts of $Y$ corresponding to the quadrilaterals $Q_{j}$ will be called the boundary pieces of $Y$. Given $\epsilon>0$, the thin part of $R$ consists of the points where the injectivity radius of is $\leq \epsilon$ and the thick part is where the injectivity radius is $>\epsilon$. The following is easy and its proof is left to the reader.

Lemma 6.1. Given $L<\infty$, there is an $\epsilon>0$ in the definition of the thick and thin parts of $R$ and $a C<\infty$, so that for any L-bounded $Y$-piece, the $\epsilon$-thin part of $R \cap Y$ is contained in the boundary pieces of $Y$ and the $\epsilon$-thick part of $R \cap Y$ is contained in a $C$-neighborhood of the central piece.

We will think of two Y-pieces as being close to each other if their boundary lengths are close, i.e., we define

$$
d\left(Y_{1}, Y_{2}\right)=\max \left(\left|\log \frac{a_{1}}{a_{2}}\right|,\left(\left|\log \frac{b_{1}}{b_{2}}\right|,\left(\left|\log \frac{c_{1}}{c_{2}}\right|\right) .\right.\right.
$$

For generalized Y-pieces we interpret $\left|\log \frac{a_{1}}{a_{2}}\right|$ as zero if $a_{1}=a_{2}=0$ and as $+\infty$ if one is zero and the other is not. Similarly for the $b$ and $c$ terms.

Lemma 6.2. Suppose $Y_{1}$ and $Y_{2}$ are two L-bounded generalized $Y$-pieces with boundary lengths $\left(a_{1}, b_{1}, c_{1}\right)$ and $\left(a_{2}, b_{1}, c_{1}\right)$ respectively. Assume that $d\left(Y_{1}, Y_{2}\right)=\epsilon=\left|\log a_{1} / a_{2}\right| \leq 2$. Then there is a $C=C(L)$ and a quasiconformal map $f: Y_{1} \rightarrow Y_{2}$ with constant $K=1+C \epsilon$ which is affine on each of the boundary components. Moreover, the Beltrami coefficient $\mu$ of $f$ is supported on the boundary piece associated to the $a_{1}$ boundary component $\gamma$ and the central part of $Y_{1}$ and it satisfies

$$
|\mu(z)| \leq C \epsilon \exp (-2 \operatorname{dist}(z, \gamma))
$$

In other words, if a is small, the corresponding dilatation decays exponentially fast away from the boundary and is zero in the thin parts (if they exist) corresponding to the two other boundary pieces.

Proof. For $Y$-pieces with three non-degenerate boundary components, the proof is immediate from the results of the previous sections (write it as a union of two symmetric hexagons, apply the previous results and use symmetry to show the map is continuous on the $Y$-piece). If there are one or more punctures on the boundary, then we obtain the result by taking limits over a sequence of non-degenerate $Y$-pieces that approach the generalized $Y$-piece. 
The following is an easy consequence which is used in the paper [6]

Lemma 6.3. Suppose $Y_{1}$ and $Y_{2}$ are two L-bounded generalized $Y$-pieces and let $D=d\left(Y_{1}, Y_{2}\right)$. Then there is a quasiconformal map $f: Y_{1} \rightarrow Y_{2}$ with constant $K=K(L, D)$ which is affine on each of the boundary components. Moreover, the dilatation $K_{f}$ of $f$ satisfies

$$
\left|K_{f}(z)\right| \leq 1+C(L, D) \exp (-2 \operatorname{dist}(z, \partial Y))
$$

\section{References}

[1] Álvarez, V. And Rodríguez, J.M.: Structure theorems for topological and Riemann surfaces. To appear in J. London. Math. Soc.

[2] Beardon, A.F.: The geometry of discrete groups. Springer-Verlag, New York, 1983.

[3] Bishop, C.J.: Non-rectifiable limit sets of dimension one. Rev. Mat. Iberoamericana 18 (2002), 653-684.

[4] Bishop, C.J.: A criterion for the failure of Ruelle's property. Preprint, 1999.

[5] Bishop, C.J.: Divergence groups have the Bowen property. Ann. of Math. 154 (2001), 205-217.

[6] Bishop, C.J.: Big deformations near infinity. Preprint, 2002.

[7] Bishop, C.J.: $\delta$-stable Fuchsian groups. Preprint, 2002.

Recibido: 29 de noviembre de 2000

Christopher J. Bishop ${ }^{1}$ Mathematics Department

SUNY at Stony Brook Stony Brook, NY 11794-3651 bishop@math.sunysb.edu

\footnotetext{
${ }^{1}$ Partially supported by NSF Grant DMS 0103626.
} 